\title{
Ingestão de plástico pelo atobá-mascarado, Sula dactylatra Lesson, 1831, na Reserva Biológica do Atol das Rocas, RN, Brasil
}

\author{
Erich de Freitas Mariano ${ }^{1 *}$ \\ Carlos Henrique Targino ${ }^{2}$ \\ ${ }^{1}$ Unidade Acadêmica de Ciências Biológicas, Centro de Saúde e Tecnologia Rural \\ Universidade Federal de Campina Grande, Campus de Patos \\ Avenida Universitária s/nº, Bairro Santa Cecília, CEP 58708-110, Patos - PB, Brasil \\ ${ }^{2}$ PPG em Ciências Biológicas, Área de concentração Zoologia \\ Universidade Federal da Paraíba, João Pessoa, PB - Brasil \\ * Autor para correspondência \\ efmariano.ufcg@gmail.com
}

Submetido em 02/09/2011

Aceito para publicação em 07/08/2012

\section{Resumo}

Partículas plásticas constituem um dos poluentes mais comuns no ambiente marinho e atingem inclusive regiões com baixa densidade populacional humana. Frequentemente, essas partículas são ingeridas por diversos organismos marinhos, ocasionando problemas no trato digestivo, que podem culminar no enfraquecimento e morte do animal. Em um estudo acerca da alimentação de 631 Atobás-mascarados, Sula dactylatra, na Reserva Biológica do Atol das Rocas, foram encontradas partículas plásticas no conteúdo estomacal de quatro indivíduos adultos. Os artefatos plásticos encontrados constituíam-se de um plástico transparente, um pedaço de rótulo de garrafa de água mineral, dois pedaços de plástico preto e um pedaço rígido pequeno e vermelho. O fluxo de embarcações, tanto pesqueiras quanto turísticas, no entorno da ReBio Atol das Rocas pode ser a fonte de origem das peças plásticas tanto nos conteúdos estomacais quanto os encontrados nas ilhas da reserva, evidenciando que ações antropogênicas já atingem áreas isoladas, as quais deveriam ter um elevado grau de proteção da biodiversidade. Diversas medidas podem ser tomadas para evitar eventos deletérios na biota marinha, porém há uma grande dificuldade em evitar o descarte de lixo proveniente de navios pesqueiros e de turismo, além dos materiais vindos do continente, sendo necessário um incremento nos esforços de conscientização e fiscalização.

Palavras-chave: Lixo marinho; Poluição; Sulidae; Unidade de conservação

\section{Abstract}

Plastic ingestion by Masked booby, Sula dactylatra Lesson, 1831, on Biological Reserve of Rocas Atoll, RN, Brazil. Plastic particles are one of the most common pollutants in the marine environment and it is reaching regions with low human population density. These particles are frequently ingested by many marine organisms, causing digestive problems, which may lead to the weakening and death of the animal. In a research on the feeding of 631-masked boobies, Sula dactylatra from the Biological Reserve of Atol das Rocas, plastic particles were found in stomach contents of four adults. The artifacts found were made of transperant plastic, a piece of label of a mineral water bottle, two pieces of black plastic and a small hard and red piece. The flow of 
fishing and tourism boats on the Atol das Rocas Biological Reserve may be the source of origin of the plastic parts in both the stomach contents and those found on the islands of the reserve, which suggests that anthropogenic behaviour has already reached isolated areas which should have a high degree of biodiversity protection. Several measures can be taken to avoid deleterious events in the marine biota, but there is great difficulty in avoiding waste dumping from fishing and tourism ships/boats, in addition to that, there are many materials from the mainland, which requires an increase in awareness and supervision.

Key words: Marine debris; Pollution; Protected areas; Sulidae

Detritos de origem antropogênica são encontrados dispersos em todos os oceanos, não apenas em áreas densamente povoadas, mas também em áreas distantes de atividades humanas (UNEP, 2009). Sheavly (2005) aponta que a natureza destes detritos tem sido alterada nos últimos 40 anos devido ao incremento de produtos sintéticos, como o plástico.

A poluição por partículas plásticas no ambiente marinho foi apontada como uma das maiores ameaças à sua biodiversidade (DERRAIK, 2002) devido à elevada capacidade de dispersão e durabilidade destes compostos plásticos (RYAN, 1987a; HANSEN, 1990; GOLDBERG, 1995; 1997). O lixo plástico é formado basicamente por resíduos de atividades pesqueiras como linhas, cabos sintéticos e redes, bem como sacos plásticos, anéis plásticos para transporte de latas (six pack rings), fibras de vidro e outros materiais que podem prender-se ou serem ingeridos por animais marinhos (BUGONI et al., 2001).

Grandes predadores marinhos, como cetáceos, tartarugas e tubarões (BUGONI et al., 2001; SAZIMA et al., 2002; MASCARENHAS et al., 2004; MEIRELLES; BARROS, 2007), são afetados pela presença do lixo plástico no ambiente, ocorrendo em muitos casos a ingestão destas partículas por serem confundidas com presas (SPEAR et al., 1995; BAIRD; HOOKER, 2000). As aves marinhas são constantemente afetadas pela incidência de plásticos no ambiente marinho, devido à sua capacidade de forrageio em extensas áreas. Desta forma servem de importantes indicadores de perturbações de origem antropogênica nas cadeias tróficas (FURNESS; CAMPHUYSEN, 1997; BURGER; GOCHFELD, 2004).

$\mathrm{Na}$ última década, tem-se aumentado o registro de ingestão de plásticos pelas aves marinhas, sendo estas partículas confundidas com presas ou ingeridas indiretamente através das presas (ROBARDS et al., 1995). A ingestão incidental ocorre no momento em que a ave forrageia e é comumente observada nas espécies que se alimentam na superfície (RYAN, 1987b), não sendo frequente nas espécies da família Sulidae, as quais mergulham para a obtenção do alimento (BEGE; PAULI, 1988; SICK, 1997).

As maiores limitações de recursos para aves marinhas dependem do controle da pesca industrial e da disponibilidade de áreas costeiras e insulares não alteradas (FURNESS, 2003; MÍNGUEZ et al., 2003) e destaca-se como um fator limitante para a conservação destas áreas o grande aporte de lixo despejado nos mares (BLIGHT; BURGER, 1997; DERRAIK, 2002). Esse problema foi ignorado por muitos anos, e apenas recentemente, vem sendo abordado por alguns autores (por exemplo, STEFATOS et al., 1999).

Nos meses de agosto e novembro de 1999, fevereiro e maio de 2000, agosto e novembro de 2002 e março e maio de 2003 foi realizado um estudo acerca da alimentação do Atobá-mascarado, Sula dactylatra Lesson, 1831, na Reserva Biológica do Atol das Rocas $\left(03^{\circ} 50^{\prime} \mathrm{S}\right.$ e $\left.33^{\circ} 49^{\prime} \mathrm{W}\right)$. Neste trabalho, foram amostrados 631 indivíduos adultos, através de lavagens estomacais, segundo metodologia proposta por Moody (1970) e detalhada por Wilson (1984).

No total amostrado $(n=631)$, foram encontradas partículas plásticas em quatro conteúdos estomacais $(0,63 \%)$ de indivíduos de $S$. dactylatra nas coletas de fevereiro de 2000 ( $1,8 \% ; \mathrm{n}=56$ conteúdos), agosto de $2002(1,9 \% ; n=104$ conteúdos) e março de 2003 (1,3\%; $\mathrm{n}=77$ conteúdos). Nos meses de agosto de 1999 ( $\mathrm{n}=$ 122), novembro de $1999(\mathrm{n}=86)$, maio de 2000 ( $\mathrm{n}=$ 50), novembro de $2000(\mathrm{n}=69)$ e maio de 2003 ( $\mathrm{n}=$ 67) foram coletados 394 conteúdos estomacais sem a presença de partículas de origem antropogênica. 
Os artefatos plásticos encontrados constituíamse de dois pedaços de plástico preto de saco de lixo, um pedaço pequeno e vermelho de plástico rígido, um plástico transparente e um pedaço de rótulo de garrafa de água mineral. Apesar da presença destes detritos nos conteúdos estomacais, os animais amostrados encontravam-se aparentemente saudáveis, apresentando comportamento de forrageio e cuidado parental normais.

A ingestão de poluentes sólidos, como os plásticos, pode ocasionar deficiências no processo digestivo, que se iniciam com o impedimento da peristalse e podem até mesmo perfurar o trato intestinal, culminando no enfraquecimento e morte do animal (LAIST, 1997).

Outros poluentes, como garrafas de água mineral, caixas de suco, latas de refrigerante e peças plásticas de motores e de embarcações, foram encontrados em grande número nas duas ilhas da Reserva Biológica do Atol das Rocas (Ilha do Farol e Ilha do Cemitério), até mesmo dentro dos ninhais, fazendo parte da constituição de ninhos (observação pessoal dos autores). Tendo em vista o idioma variado (por exemplo, inglês e árabe) de alguns dos rótulos encontrados podemos inferir que estes poluentes chegaram às ilhas através das correntes marinhas ou mesmo que sua procedência seja de navios de cruzeiros turísticos os quais têm suas rotas dentro da área protegida. Barcos pesqueiros provenientes da frota artesanal do Rio Grande do Norte, Paraíba e Ceará são frequentemente observados no local, quando estão em rota para seus pontos de pesca ou mesmo operando de forma ilegal. Esta presença favorece o aparecimento nas ilhas de diversos artefatos pesqueiros como tonéis de plástico, boias de isopor, além de redes de emalhe que ficam presas nos corais e são deixadas para trás. A visitação na reserva é restrita às atividades de pesquisa e todo o lixo produzido e encontrado em cada expedição é cuidadosamente armazenado para retorno ao continente.

Apesar da presença de lixo plástico em conteúdos estomacais de aves marinhas já ter sido citada na literatura, a ingestão de plástico por $S$. dactylatra no Brasil ainda não havia sido relatada. Estudos de alimentação no Brasil com S. leucogaster (BOTH; FREITAS, 2001; BRAUN, 2002; BRANCO et al., 2005; MARIANO, 2005) e $S$. sula (dados não publicados), incluindo no Atol das Rocas, também não apresentaram registros de lixo. Entretanto, a ocorrência de lixo em ninhos de atobás já foi evidenciada para Morus bassanus, em colônias do Noroeste Atlântico (MONTEVECCHI, 1991), inclusive com uma pequena porcentagem de óbitos de adultos e ninhegos causada pelo emalhe em materiais sintéticos incorporados aos ninhos.

As atividades e políticas de preservação voltadas para as aves marinhas esbarram na complexidade do modo de vida desses animais, tendo em vista que uma única espécie pode utilizar um amplo espectro de habitats e que sua área de ocorrência ultrapassa os limites das fronteiras políticas (MÍNGUEZ et al., 2003). Complicando ainda mais esse fato, temos medidas políticas dispersas, inúmeras complicações administrativas e medidas de manejo adotadas sem cooperação entre os governos.

Estratégias para a conservação, adotadas pela BirdLife International, baseiam-se em quatro conceitos (pilares): espécies, localidades, habitats e pessoas. Estes "pilares da conservação" ajudam-nos a identificar as prioridades para o manejo das aves em nível global (GALLO-ORSI, 2003). Entre esses pilares encontramos as pessoas, as quais são os principais responsáveis para que o processo de manejo e conservação funcione; e para a implementação desta ação de manejo são necessárias decisões burocráticas das agências de meio ambiente e, consequentemente, tempo.

Inúmeras medidas podem ser tomadas para evitar a poluição por plásticos na biota marinha em áreas afastadas de centros populacionais, porém há uma grande dificuldade em evitar o descarte do lixo proveniente de navios pesqueiros e de turismo, além dos materiais vindos do continente nestas áreas mais remotas, sendo necessários altos investimentos para ações de conscientização e fiscalização.

A presença de partículas plásticas em conteúdos estomacais das aves ocorrentes na Reserva Biológica do Atol das Rocas e nas próprias ilhas contidas na reserva mostra que ações antropogênicas indiretas estão afetando áreas isoladas, que deveriam ter um elevado grau de proteção para a preservação da vida selvagem. 


\section{Referências}

BAIRD, R. W.; HOOKER, S. K. Ingestion of plastic and unusual prey by a juvenile Harbour Porpoise. Marine Pollution Bulletin, Amsterdam, v. 40, p. 719-720, 2000.

BEGE, L. A. R.; PAULI, B. T. As aves nas Ilhas Moleques do Sul - Santa Catarina: aspectos da ecologia, etologia e anilhamento de aves marinhas. Florianópolis: Fundação do Meio Ambiente (FATMA), 1988. 64 p.

BLIGHT, L. K.; BURGER, A. E. Occurrence of plastic particles in seabirds from the Eastern North Pacific. Marine Pollution Bulletin, Amsterdam, v. 34, p. 323-325, 1997.

BOTH, R.; FREITAS, T. R. O. Dieta do atobá-marrom (Sula leucogaster), da viuvinha-marrom (Anous stolidus) e da viuvinhanegra (Anous minutus) no arquipélago de São Pedro e São Paulo, Brasil. In: ALBUQUERQUE, J. L. B.; CÂNDIDO, J. F.; STRAUBE, JR. F. C.; ROOS A. L. (Ed.). Onitologia e conservação: da ciência às estratégias. Tubarão: UNISUL, 2001. p. 313-326.

BRANCO, J. O.; FRACASSO, H. A. A.; MACHADO, I. F.; BOVENDORP, M. S.; VERANI, J. R. Dieta de Sula leucogaster Boddaert (Sulidae, Aves), nas Ilhas Moleques do Sul, Florianópolis, Santa Catarina, Brasil. Revista Brasileira de Zoologia, Curitiba, v. 22, n. 4, p. 1044-1049, 2005.

BRAUN, J. R. R. Aspectos da bioecologia do Atobá-marrom Sula leucogaster (Sulidae, Ave), nas Ilhas Moleques do Sul, Florianópolis, SC. 2002.45 f. Monografia (Curso de Oceanografia) - Universidade do Vale do Itajaí, Itajaí. 2002.

BUGONI, L.; KRAUSE, L.; PETRY, M. V. Marine debris and human impact on sea turtles in southern Brazil. Marine Pollution Bulletin, Amsterdam, v. 42, p. 1330-1334, 2001.

BURGER, J; GOCHFELD, M. Mercury in canned tuna: white versus light and temporal variation. Environmental Research, Montreal, v. 96, n. 2, p. 239-249, 2004.

DERRAIK, J. G. B. The pollution of the marine environment by plastic debris: a review. Marine Pollution Bulletin, Amsterdam, v. 44, p. 842-852, 2002.

FURNESS, R.W. Impacts of fisheries on seabird communities. Scientia Marina, Barcelona, v. 67, n. 2, p. 33-45, 2003.

FURNESS, R. W.; CAMPHUYSEN, C. J. Seabirds as monitors of the marine environment. ICES Journal of Marine Science, Copenhagen, v. 54, p. 726-736, 1997.

GALLO-ORSI, U. Species action plans for the conservation of seabird in the Mediterranean Sea: Audouin's gull, Balearic shearwater and Mediterranean shag. Scientia Marina, Barcelona, v. 67 , n. 2, p. 47-55, 2003.

GOLDBERG, E. D. The health of the oceans - a 1994 update. Chemistry and Ecology, Amsterdam, v. 10, p. 3-8, 1995.

GOLDBERG, E. D. Plasticizing the seafloor: an overview. Environmental Technology, London, v. 18, p. 195-202, 1997.

HANSEN, J. Draft position statement on plastic debris in marine environments. Fisheries, Bethesda, v. 15, p. 16-17, 1990.

LAIST, D. W. Impacts of marine debris: entanglement of marine life in marine debris including a comprehensive list of species with entangled and ingestion records. In: COE, J. M.; ROGERS, D. B. (Ed.). Marine debris: sources, impacts, and solutions. New York: Springer-Verlag, 1997. p. 9-139.
MARIANO, E. F. Análise de conteúdos estomacais de Sula leucogaster (Boddaert, 1783) (Pelecaniformes, Sulidae) do Atol das Rocas, RN. 2005. 70 f. Monografia (Bacharelado em Ciências Biológicas) - Universidade Federal da Paraíba, João Pessoa. 2005.

MASCARENHAS, R.; SANTOS, R.; ZEPPELINI, D. Plastic debris ingestion by sea turtles in Paraíba, Brazil. Marine Pollution Bulletin, Amsterdam, v. 49, p. 354-355, 2004.

MEIRELleS, A. C. O.; BARROS, H. M. D. R. Plastic debris ingested by a rough-toothed dolphin, Steno bredanensis, stranded alive in northeastern Brazil. Biotemas, Florianópolis, v. 20, n. 1, p. 127-131, 2007.

MÍNGUEZ, E.; ORO, D.; De JUANA, E.; MARTÍNEZ-ABRAÍN, A. Mediterranean seabird conservation: what can we do? Scientia Marina, Barcelona, v. 67, n. 2, p. 3-6, 2003.

MONTEVECCHI, W. A. Incidence and types of plastic in gannets' nests in the northwest Atlantic. Canadian Journal of Zoology, Ottawa, v. 69, n. 2, p. 295-297, 1991.

MOODY, T. A method for obtaining food samples from insectivorous birds. Auk, Albuquerque, v. 87, p. 579, 1970.

ROBARDS, M. D.; PIATT, J. F.; WOHL, K. D. Increasing frequency of plastic particles ingested by seabirds in the Subarctic North Pacific. Marine Pollution Bulletin, Amsterdam, v. 30, p. 151-157, 1995.

RYAN, P. G. The origin and fate of artefacts stranded on islands in the African sector of the Southern Ocean. Environmental Conservation, Cambridge, v. 14, p. 341-346, 1987 a.

RYAN, P. G. The incidence and characteristics of plastic particles ingested by seabirds. Marine Environmental Research, Ancona, v. 23 , p. $175-206,1987 b$.

SAZIMA, I.; GADIG, O. B. F.; NAMORA, R. C.; MOTTA, F. S. Plastic debris collars on juveniles carcharhinid sharks (Rhizoprionodon lalandii) in southwest Atlantic. Marine Pollution Bulletin, Amsterdam, v. 44, p. 1147-1149, 2002.

SHEAVLY, S. B. Marines debris - an overview of a critical issue for our ocean. In: MEETING OF THE UN OPEN-ENDED INFORMAL CONSULTATIVE PROCESS ON OCEANS \& THE LAW OF THE SEA, 7, 2005, New York. Procedings... New York, 2005. Versão eletrônica.

SICK, H. Ornitologia brasileira. Rio de Janeiro: Editora Nova Fronteira, 1997. $862 \mathrm{p}$.

SPEAR, L. B.; AINLEY, D. G.; RIBIC, C. A. Incidence of plastic in seabirds from the Tropical Pacific, 1984-91: relation with distribution of species, sex, age, season, year and body weight. Marine Environmental Research, Ancona, v. 40, n. 2, p. 123-146, 1995.

STEFATOS, A.; CHARALAMPAKIS, M.; PAPATHEODOROU, G.; FERENTINOS, G. Marine debris on the seafloor of the Mediterranean Sea: examples from two enclosed gulfs in Western Greece. Marine Pollution Bulletin, Amsterdam, v. 36, p. 389-393, 1999.

UNEP - UNITED NATIONS ENVIRONMENT PROGRAMM. Marine litter: a global challenge. Nairobi: UNEP, 2009. 232 p.

WILSON, R. P. An improved stomach pump for penguins and other seabirds. Journal of Field Ornithology, Houston, v. 55, p. 109$112,1984$. 